\title{
Funneling and Saltation Effects for Tactile Interaction with "Detached" Out of the Body Virtual Objects
}

\author{
Jaedong Lee, Sangyong Lee, and Gerard J. Kim* \\ Digital Experience Laboratory, \\ Korea University, Seoul, Korea \\ \{jdlee, xyleez,gjkim\}@korea.ac.kr
}

\begin{abstract}
In a previous work, we confirmed the existing effects of "Out of the Body" tactile illusion for virtual and augmented objects through funneling and saltation. However, it required a virtual imagery to be attached to the user for directly extending one's body. This paper aims at investigating similar phantom tactile sensations exist when the virtual object is visually detached from the user's body. Two usability experiments were conducted to verify the hypothesized phantom tactile effects: one for funneling and the other, saltation. Our results have shown that in addition to the perception of the phantom sensations with the "detached" visual feedback, the interaction experience was significantly enriched (vs. when without explicit visual feedback). We also discovered for the first time that for funneling, phantom sensations can be elicited without any visual feedback at all. The findings can be applied to the tactile interaction design using minimal number of actuators on a variety of media platforms including the mobile, holography and augmented reality.
\end{abstract}

Keywords: Phantom sensation, Illusory feedback, Funneling, Saltation, Vibro-tactile feedback, Multimodal feedback.

\section{Introduction}

Tactile feedback has become almost indispensable in improving interaction experience. Realization of tactile feedback by using vibration devices is one inexpensive and practical method. However, due to its size and mechanics, a single vibrator scheme is most often employed and it is only able to convey simplistic on-off type of events. Instead, as a way to improve the tactile experience, a more advanced form of vibro-tactile feedback most often involves an array of vibrators [6, 7] that brings about mechanical and cost complications and a constraint that a relatively significant area of the body has to be in full contact with the array.

One way that researchers have considered to overcome this problem is to create illusory (or pseudo) tactile feedback and combining it with the corresponding visual or auditory feedback [19]. For example, in a previous work, we confirmed the existing effects of "Out of the Body" tactile illusion for virtual and augmented objects through

\footnotetext{
* Corresponding author.
} 
saltation and funneling. However, it required a virtual imagery to be attached to the user for directly extending and connecting one's body. In other words, this is undesirable because the user must have both hands/fingers in contact with the virtual object (see Figure 1). Thus, this paper aims at reproducing the same "Out of the Body" phantom sensation effect with "floating" (i.e. no virtual extension) dynamic virtual object detached from the body (see Figure 3). In particular, we consider two phantom tactile sensation phenomena, namely funneling [4] and saltation [14].

Funneling (Saltation) refers to the illusory tactile sensation occurring away from the actual places of timed (simultaneous) vibratory stimulations. The intended location of the phantom sensation can be changed by modulating the intensity (Funneling) or inter-stimulus time interval (Saltation). Funneling and saltation have often been applied to reduce the number of tactile actuators in tactile interaction design $[18,35]$. Recently, researchers have discovered such phantom sensations can be extended to the "Out of the Body" [27] and for "Out of the Body" virtual objects [24] (see Figure 1), thus making it possible to generate phantom tactile sensations as if coming from an external object (both real and virtual). Possible applications of such a phenomenon are shown in Figure 2.

Seen by a special display such as an HMD
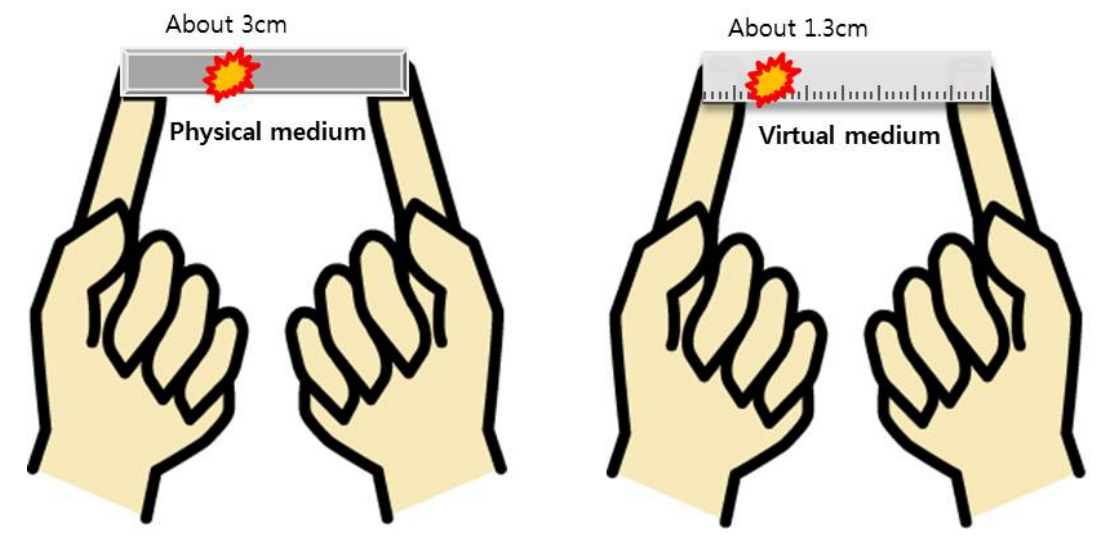

Fig. 1. The concept of "Out of the Body" tactile experience from a hand-held physical or virtual medium. A medium for extending and connecting the body parts is required, virtual or real. The phantom sensation is more evident with the physical medium (e.g. felt at $3 \mathrm{~cm}$ from the left) than the virtual (felt at $1.3 \mathrm{~cm}$ with the same stimulation) [24].

It has been found that the extent or the controllability of the effect is diminished when extended to "Out of the Body" and even more so when a virtual object is used as the medium extending one's body [24, 27]. Consequently, we seek and experimentally investigate the possible synergistic effects by associating it with "dynamic" visual feedback to improve the tactile experience and controllability, possibly even without the medium (real or virtual) that connects the body parts (i.e. tactile interaction with virtual objects completely detached from the body). If validated, such a phenomenon can be applied to tactically interacting with holographic objects hovering in the air (Figure 2(b)). 


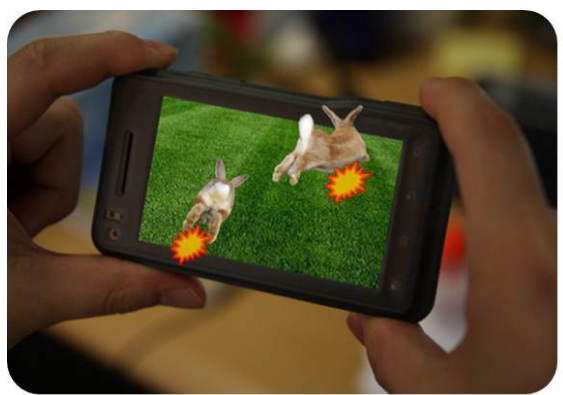

(a)

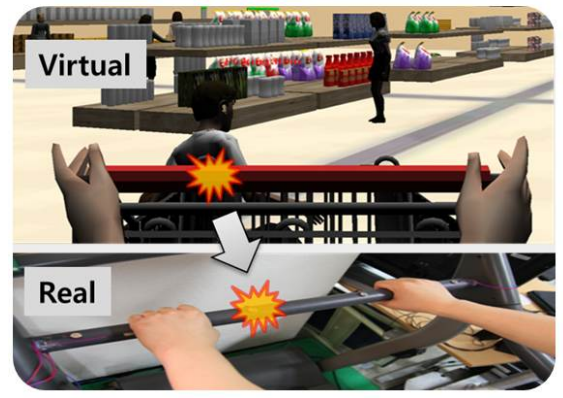

(c)

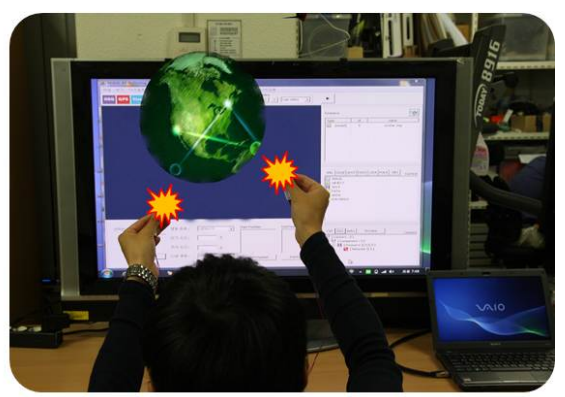

(b)

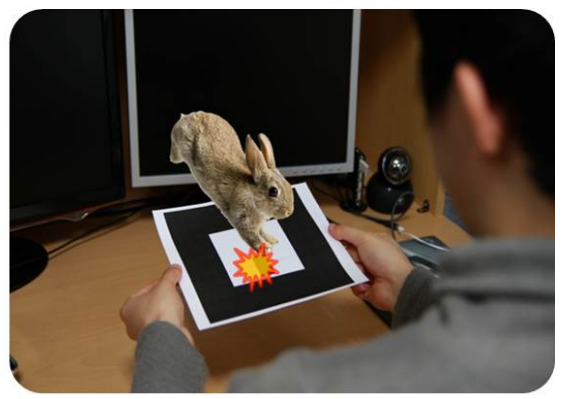

(d)

Fig. 2. Possible applications of the "Out of the Body" phantom tactile sensation: Two handed/fingered interaction and feeling tactile sensations as if coming from the middle of the (a) mobile device, (b) hovering holographic-virtual imagery, (c) indirectly from a virtual object in a monitor and (d) an augmented marker (e.g. seen through a head mounted display)

This paper is organized as follows. In the next section, we first review previous research literatures related to phantom tactile sensation, multi-sensory integration and their application to practical interaction design. Then, we describe the two validation experiments and report the results. Finally, we conclude the paper with a discussion and directions for future research.

\section{Related Work}

Funneling and saltation are the two major perceptual illusion techniques for vibrotactile feedback. Funneling refers to stimulating the skin at two (or more) different locations simultaneously with different amplitudes and eliciting phantom sensations in the space between [1,4]. Several researchers have applied this phenomenon to human interfaces [3, 19, 28, 31, 32]. For instance, Hoggan et al. experimented with using three vibrators on a mobile device to emulate a tactile progress indicator [18]. Tan et al. applied saltation to implement a tactile chair using a 3 x 3 tactile array for a pattern recognition application [38]. 
Miyazaki has discovered the saltation could be extended to body-worn (e.g. handheld) objects and to create "Out of the Body" tactile experience [27]. Furthermore, Lee et al. has confirmed the same phenomenon existed for virtual objects but with reduced effects and less precise controllability [24]. Other researchers have investigated different ways and effects to apply phantom sensation by employing different stimulation interpolation methods [1], varying the values of ISI's $[10,15,16]$, relative vibration amplitudes [1, 31], stimulation duration and frequency [10, 37], interstimulation distances [32], and even applying saltation to non-continuous skin (e.g. from the right arm to the left, fingertip to fingertip) [12, 40]. Note that in our previous work, only minimal static visual feedback was used, namely the virtual "ruler" representing only the medium bridging the two body points (rather than the actual visual representation of the tactile event) at which the actual vibratory stimulations were given, to recreate the original "Out of the Body" phenomenon. No detailed studies have been reported regarding phantom tactile feedback with dynamic or detached visual feedback. Also note that aforementioned works $[3,18,19,28,31,32$, 38] that have applied tactile phantom sensations to human interfaces did it so directly to the human skin and had not investigated the use of the "Out of the Body" phenomenon nor the issue of thereby minimizing the number of tactile actuators.

Interestingly, Flach et al. [13] and Kilgard [21] have found that the phantom sensation was much influenced by the subject's focus of attention, anticipation and/or the line of sight. This strongly hints the possibility of further synergistic effects with more apparent visual effects associated with the intended phantom sensation.

In fact, the synergistic sensory integration is not new. It is generally accepted that multi-sensory feedback is additively helpful to interactive task performance [23]. This is only true provided when the respective modality feedback is consistent in its content and timing with one another [30]. Many synergistic multimodal interaction systems have been devised and studied employing gestures [5], voice [11], proprioception [26], speech/audio [17], and force feedback [34]. Aside from just improving task performance, multisensory interactions can also modify user perception, as illustrated by the famous McGurk effect. The McGurk effect is a perceptual phenomenon in which vision alters speech perception [25]. Simple visual tricks can easily alter the body image that is created by the proprioceptive sense [33]. Although the best known cross modal effects are those of vision influencing other modalities, visual perception can be altered by other modalities as well [39].

\section{Experiment I: Effects of Funneling with "Detached" Visual Object}

\subsection{Purpose and Hypothesis}

In the first experiment, we have compared the tactile experience of funneling for a virtual object, between (1) when it was associated with a (dynamic) visual presentation detached from the body and (2) when no visual presentation is given at all (as a reference). In a usual application setting, virtual objects will normally be rendered 
without a part that visually extends or connects user's body parts (Figure 2). Our interest is first to assess whether funneling elicits phantom sensation, its extent and effects to the overall interaction experience.

It is well expected that no dislocated phantom tactile experience will be elicited without any visual feedback (Figure 4, bottom right). We still test for it as a base case. Also it has been shown through prior research [27] that singular vibration (e.g. with a single vibrator) cannot create any localized phantom sensation for "virtual" object external to the body either. To reiterate, we are interested in and hypothesize the existence and quality of the phantom tactile sensation of the virtual objects even when it does not directly attached to the user body. We also expect that results for the detached "Out of the Body" virtual objects with the hypothesis that results will mostly extend to detached "Out of the Body" physical objects

\subsection{Experimental Design and Set Up}

To create the funneling based phantom sensation, the user was given simultaneous tactile stimulations to one's two index fingers, one in the right and the other in the left. The two fingertips were tracked using small markers $(25 \mathrm{~mm} \times 25 \mathrm{~mm})$ by a head mounted camera and a "detached" augmented reality video imagery was presented to the user through a 47 inch monitor (nominal viewing distance: $\sim 60 \mathrm{~cm}$ ). As for the augmented visual feedback, a small moving object (“a bouncing basketball”) was rendered at the intended location of sensation between the two fingers (see Figure 3).

The OSGART [29] was used to recognize/track the small markers and generate the augmented video imagery. A webcam was worn on the head mounted fixture to produce a view close to one according to the actual line of sight. The user was asked to maintain a nominal distance $(8 \mathrm{~cm})$ between the fingers (using an $8 \mathrm{~cm}$ wide marking on the table), but was allowed small movements for natural and comfortable interaction. The inter-finger distance of $8 \mathrm{~cm}$ was used and set equal to the experimental conditions used in [24, 27].

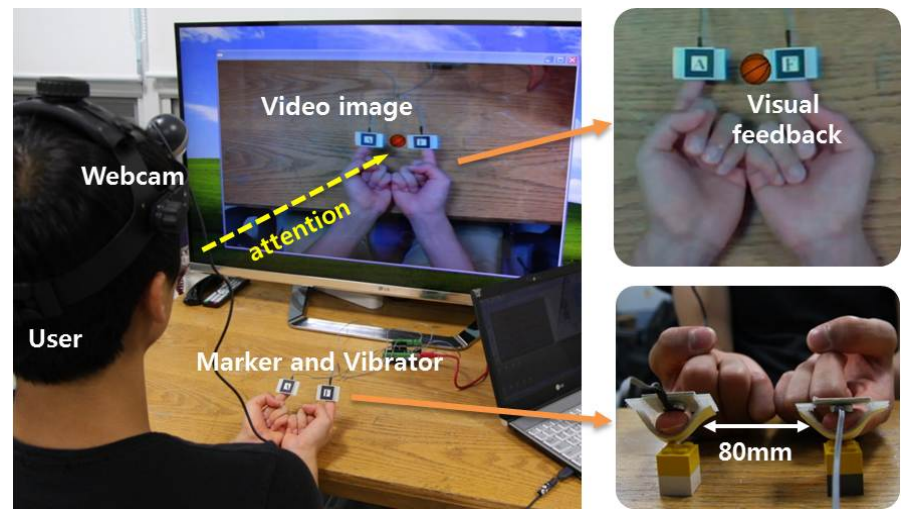

Fig. 3. The experimental set up for Experiments I and II. Vibratory stimulations were given to the two index fingers and the augmented visual feedback (e.g. bouncing basketball), detached from the fingers, seen through a large display monitor. 
A common flat coin-type vibrator (11 mm in diameter) was used (taped to the respective fingertips) and controlled by an Arduino board [2] (and interfaced to and synchronized with the OSGART based experiment software). For detailed specifications refer to [20]. It is controlled by a voltage input using a pulse width modulation signal with an amplitude between 0 to $5 \mathrm{~V}$, which in turn produces vibrations with frequency between 0 and $250 \mathrm{~Hz}$ and associated amplitudes between 0 to $2 \mathrm{G}$ (measured in acceleration, or 0 to $18 \mu \mathrm{m}$ in position) respectively. According to [20,36], these values are well above the human's normal detection threshold (about $6 \sim 45 \mathrm{db}$ ).

The experiment was designed as a $2 \times 5$ factor within-subject. The two factors were (1) inclusion of the visual feedback (with or without), and (2) intended locations of tactile illusion (five locations between the fingers labeled P1 P5). Four survey questions were answered in a 7 Likert scale asking of the various aspects of the phantom tactile experience.

\subsection{Detailed Procedure}

Twenty paid subjects ( 15 men and 5 women) participated in the experiment with the mean age of 25.5. After collecting one's basic background information, the subject was briefed about the purpose of the experiment and instructions for the experimental task. A short training ( 3 minutes) was given for the subject to get familiarized to the experimental process. In addition to the head mounted fixture for the camera, the subjects wore ear muffs to prevent any bias from the sounds of the vibration. The ear muff was tested to make sure so that no sound could be heard during the experiment, and did not affect the outcome of the experiment.

The levels of stimulations were given with the intention to create phantom and real sensations at 5 equi-distanced locations between two fingers. The Linear variation of stimulus amplitudes methods of Alles [1] (Figure 4) was used with the stimulation duration set at $200 \mathrm{~ms}$. Preliminary studies and prior research has also confirmed that the aforementioned linear method and stimulation duration exhibited the best effect [24]. The visual feedback appeared at the intended location of sensation, at the time of the stimulations, stayed for 200 milliseconds (same as the tactile stimulation duration) and disappeared.

Each subject experienced, in a balanced order, a total of 60 positional feedbacks in all the 2 x 5 (10) conditions (6 repetitions each) with 10 second inter-stimulus rest interval, lasting about 40 minutes. For each condition, two exact same stimulation patterns were given, then subjects were asked to indicate the place of phantom sensations in terms of the five prescribed positions. We use the symbols L1 L5 to indicate the subject's response (as distinguished from the actual intended locations of sensations). The subjects were explicitly asked to report the place of tactile (e.g. rather than visual) sensation right after experiencing the stimulation. In addition, after all trials, they were asked to answer a short survey about their subject feelings (questions shown in Table 1). 

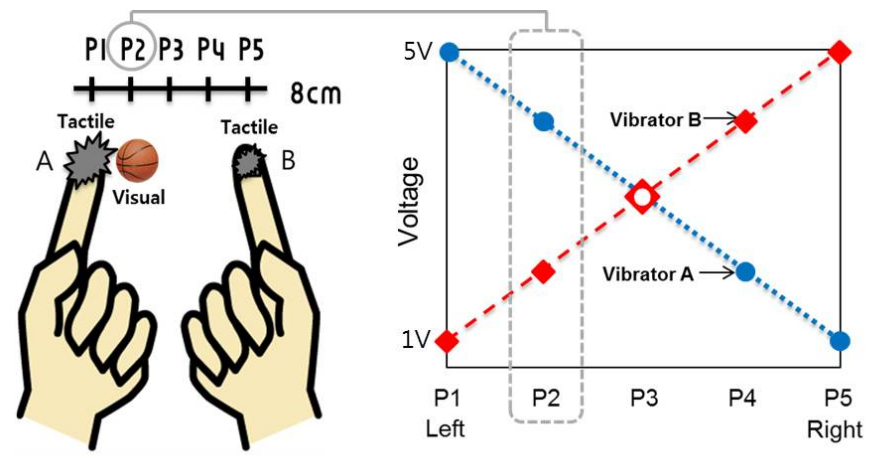
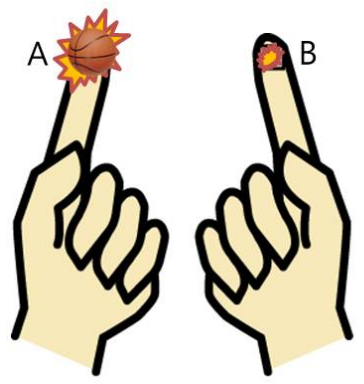

Rendering P1 (Visual feedback)

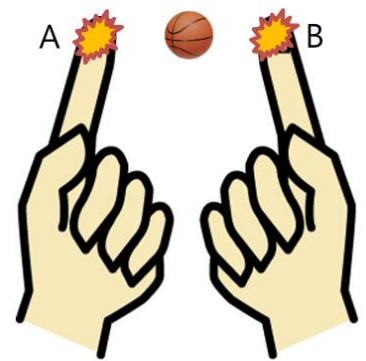

Rendering P3 (Visual feedback)
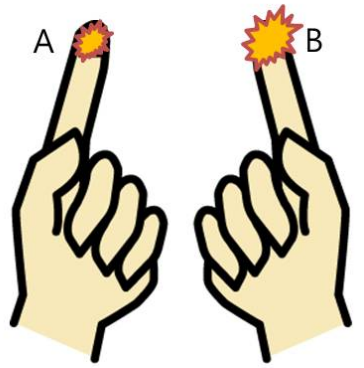

Rendering P4

(Non-visual feedback)

Fig. 4. The rendering method of stimulation originally proposed by Alles [1] for funneling to generate phantom sensations at five different positions (top). Examples of tactile stimulation: at P1 and P3 with visual feedback (bottom left and middle) and at P4 without visual feedback (bottom right). For example, to produce a phantom sensation at P2, a simultaneous stimulation of $4 \mathrm{~V}$ at $\mathrm{A}$ and $2 \mathrm{~V}$ at $\mathrm{B}$ are given as shown in the top left.

Table 1. The four survey questions regarding the subjective feel for the phantom sensation answered in 7 Likert scale

\begin{tabular}{|l|l|}
\hline Q1 & $\begin{array}{l}\text { Were you able to perceive phantom sensation? } \\
(1: \text { Not at all } \sim 7: \text { Very well })\end{array}$ \\
\hline Q2 & $\begin{array}{l}\text { When you perceive phantom sensation, did visual feedback affect you? } \\
(1: \text { Not at all } \sim 7: \text { Very much })\end{array}$ \\
\hline Q3 & $\begin{array}{l}\text { How confidence are you about your answer to Q1? } \\
(1: \text { Not confident at all } \sim 7: \text { Very confident })\end{array}$ \\
\hline Q4 & $\begin{array}{l}\text { How long did it take you to perceive the phantom sensation if any? } \\
(1: \text { Instantly } ~ 7: \text { Few seconds })\end{array}$ \\
\hline
\end{tabular}

\subsection{Results}

Figure 5(a) and (b) each shows the sensed/perceived locations of the tactile sensation (vertical axis), elicited by funneling, as reported by the users vs. the intended 
locations (horizontal axis) of sensation with visual effects and without. To our surprise, even without a virtually mediating object, phantom sensations were perceived at all five intended locations (Figure 5(b)). This is a first time discovery to our knowledge. There is still clear marked difference in the accuracy (or variance) for the intermediate locations, P2 P4. Note that P1 and P5 are where the vibrators are actually located, thus, a correct perception even without visual effect is naturally expected. Also note that the perceived locations were different among each other with statistical significances (see Table 2). Thus, a high localization controllability $(\sim \pm 4 \mathrm{~mm})$ at approximately $2 \mathrm{~cm}$ resolution was possible. Consistently to the statistical results, subjects reported that when no visual effects were given, it was difficult to differentiate between P1 and P2 (and similarly for P4 and P5), where the vibration motors were actually placed.

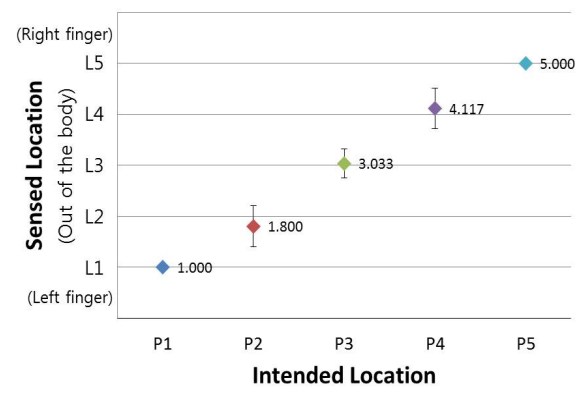

(a) With the associated visual effects

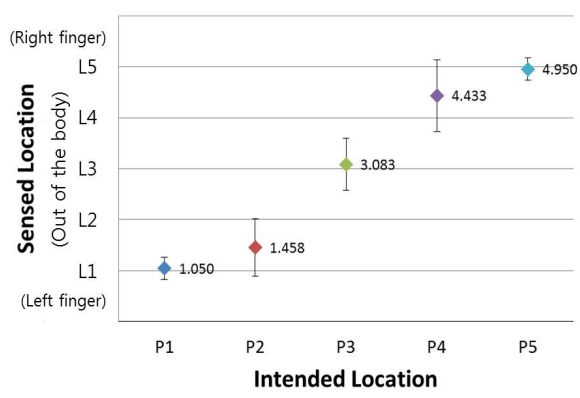

(b) Without visual effects

Fig. 5. Accuracy of reported locations of the phantom sensation with respect to the intended: (a) with associated visual effect and (b) without

Table 2. Statistical differences (p-values) in the perceived locations (L1 L5)

\begin{tabular}{l||l|l|l|l}
\hline & L1-L2 & L2-L3 & L3-L4 & L4-L5 \\
\hline With visual & $<\mathbf{0 . 0 0 1}$ & $<\mathbf{0 . 0 0 1}$ & $<\mathbf{0 . 0 0 1}$ & $<\mathbf{0 . 0 0 1}$ \\
Without & $<\mathbf{0 . 0 0 1}$ & $<\mathbf{0 . 0 0 1}$ & $<\mathbf{0 . 0 0 1}$ & $<\mathbf{0 . 0 0 1}$ \\
\hline
\end{tabular}

ANOVA revealed statistically significant differences existed in the senses locations between when the visual effect was given and when it was not, at all five locations except at the middle, L3 (Figure 6 and Table 3). Note that with funneling, when equal stimulation strengths are given at the two finger tips and it is plausible to think that it would be easier (that is, no help needed with the visual effect) to perceive the phantom sensation to come from the middle and make the proper response. 


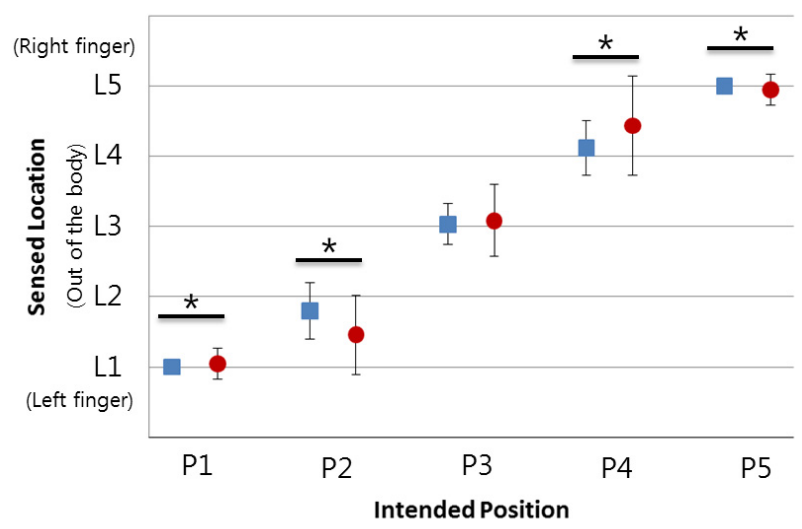

Fig. 6. A pair-wise comparison of the perceived locations between when visual effect is given (square) and not (circle). Star marks indicate those with statistically significant differences.

Table 3. Statistical differences (p-values) in the "differences" of perceived locations between when with associated visual effect (e.g. L1) and without (L1')

\begin{tabular}{l||l|l|l|l}
\hline L1-L1' & L2-L2' & L3-L3, & L4-L4' & L5-L5, \\
\hline $\mathbf{0 . 0 1 3}$ & $<\mathbf{0 . 0 0 1}$ & 0.352 & $<\mathbf{0 . 0 0 1}$ & $\mathbf{0 . 0 1 3}$ \\
\hline
\end{tabular}

Figure 7 shows the number of correct answers (i.e. correct when the perceived location matches the intended within a pre-specified threshold) in terms of score out of 100. Similarly to the above analysis, performances were generally worse without the associated visual effects for intermediate locations P2 and P4 being confused with

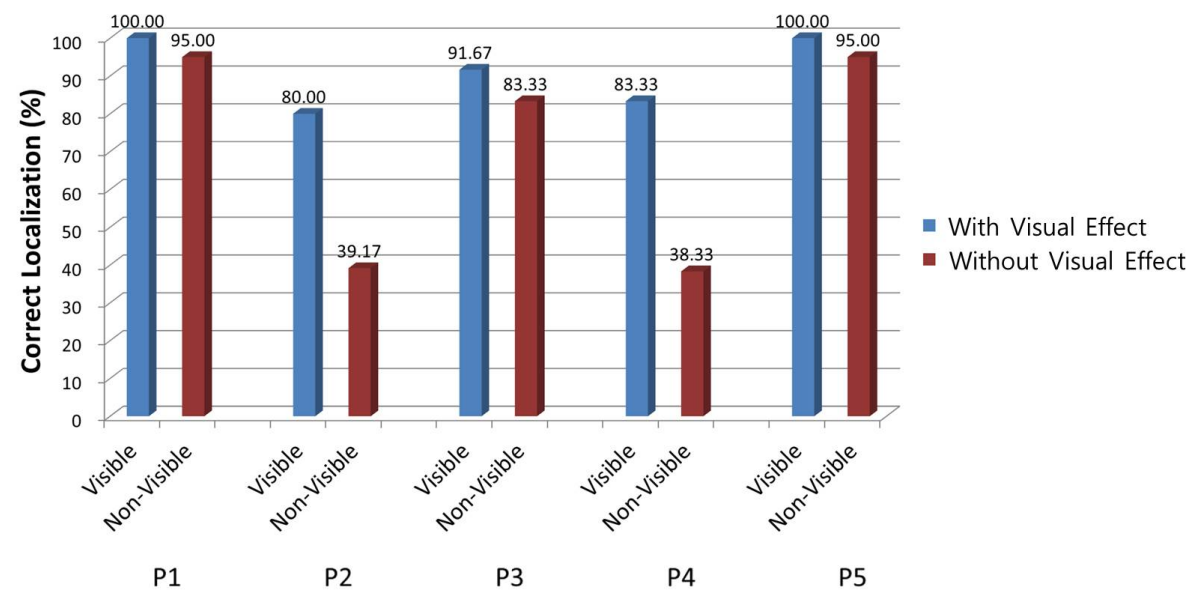

Fig. 7. Comparison of correct localization of visual and non-visual feedback based on stimulations across intended position 
P1 and P5 respectively. We emphasize that while distinguishing of five distinct points within the $8 \mathrm{~cm}$ distance was possible with visual feedback or without, the accuracy (how close the perceived is to the intended location of sensation) is expectedly lower when the visual feedback is absent.

Finally, Figure 8 shows the responses to the four survey questions, which are mostly consistent with the quantitative analysis. Subjects were conscious of the helpful effects of the visual feedback and confident of their phantom sensations.

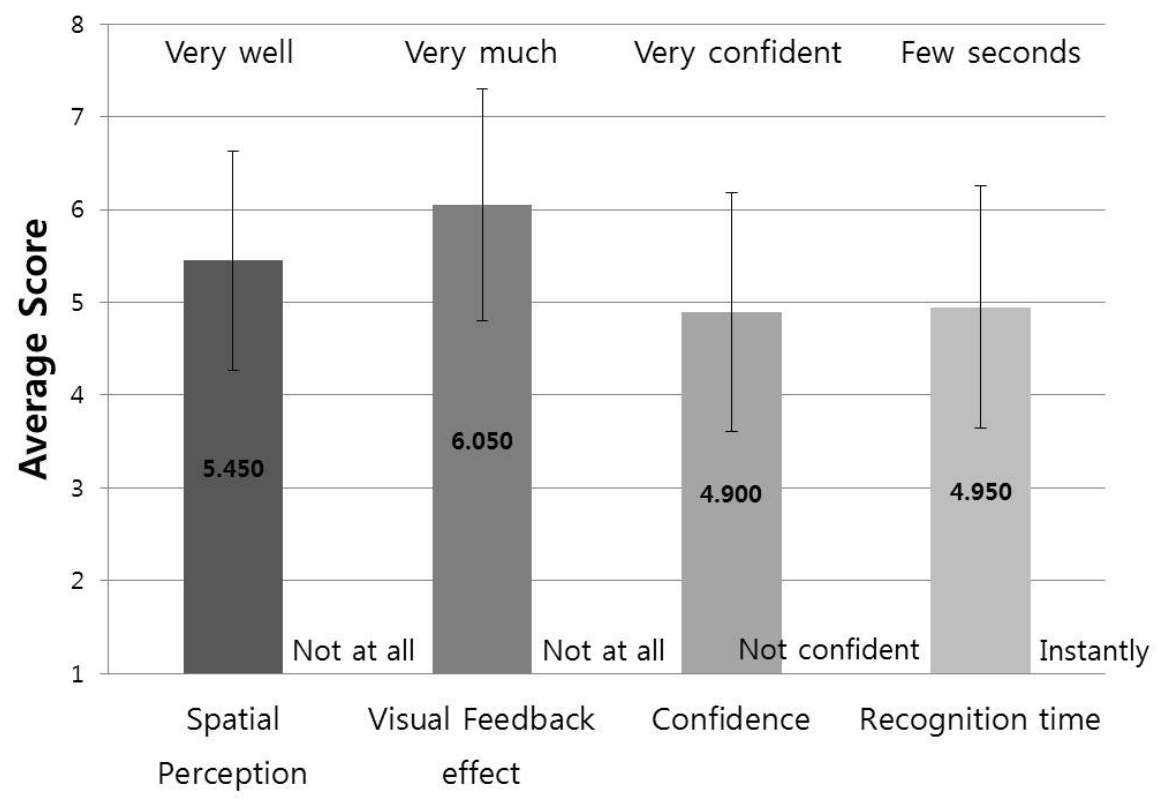

Fig. 8. The collective responses to the general usability/experience survey

\section{Experiment II: Effects of Saltation with "Detached" Visual Object}

\subsection{Purpose and Hypothesis}

The second experiment is mostly similar to the first except that the "detached" visual feedback effect to saltation was tested instead. We hypothesize for the existence and the improved quality of the phantom tactile sensation of the virtual objects when coupled with visual effects.

\subsection{Experimental Design and Set Up}

Again the basic experimental design and set up is mostly identical to the first one. Since saltation was used the vibro-tactile stimulations were timed rather than given simultaneously. The same "detached" augmented visual feedback, "bouncing 
basketball" was used. The experiment was designed as a $2 \times 5 \times 2$ factor within-subject. The three factors were (1) inclusion of the visual feedback (with or without), (2) intended locations of tactile illusion (five locations between the fingers labeled P1 P5) and (3) direction of the stimulation (from right to left or vice versa). Five survey questions were answered in a 7 Likert scale asking of the various aspects of the phantom tactile experience (see Table 4).

\subsection{Detailed Procedure}

Twenty paid subjects ( 15 men and 5 women) participated in the experiment with the mean age of 25.2 (a different pool from Experiment I). The experimental procedure was mostly identical to the first. Thus, we only describe the way saltation (i.e. timed stimulation) was administered in the treatments. Figure 9 pictorially describes how the timed vibro-tactile stimulations were given to create saltation effects. A total of three consecutive stimulations were given, each labeled S1, S2 and S3. The first two stimulation were given at P1 and the third at P5, intending to create a phantom sensation somewhere between P1 and P5. Inter-stimulus intervals of S1-S2 and S2-S3 were given with the intention to create phantom sensations at the prescribed positions, namely, P1 P5 (800ms-50ms) respectively (and stimulation duration of 80ms) based on recommended values for best effects from prior research and our own previous experiments [24].

Half of the saltation treatments were administered with right to left stimulations (at P5, then P1) and the other half, in the opposite directions (at P1, then P5). The visual effects were rendered at P1, P5 (where the actual stimulations were given) and at the intended location of sensation and stayed on for 80 milliseconds, same as the stimulation duration. As shown in Figure 10, the visual feedback was given, synchronized with the corresponding of the three tactile stimulations $(\mathrm{S} 1 \sim \mathrm{S} 3)$, at three locations (two at the finger tips where the actual stimulations are given and one in between at the intended location of sensation, one of P's). We stress again that the users were asked of the location and quality of the tactile feedback, not the visual.

Each subject experienced, in a balanced order, a total of 72 positional feedbacks in all the conditions: 60 times with visual feedback ( 2 directions, 5 intended locations of
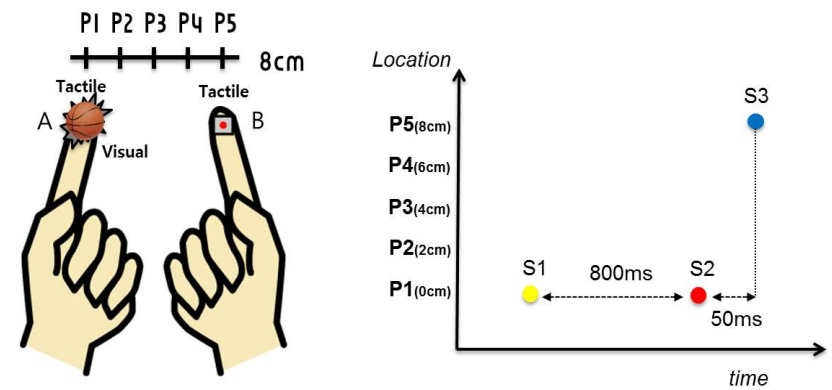

Fig. 9. The rendering method for saltation stimulation. Three timed stimulations of S1 (at P1), $\mathrm{S} 2$ (at P1) and S3 (at P5). 

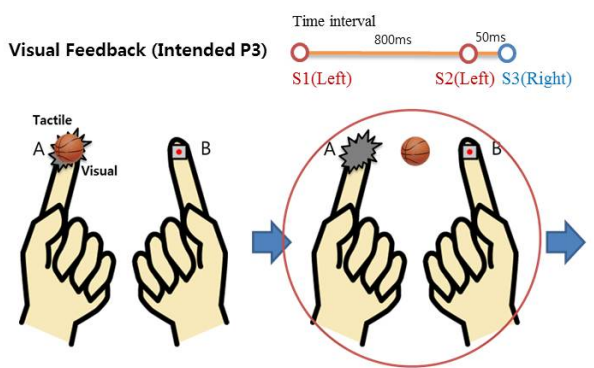

S1
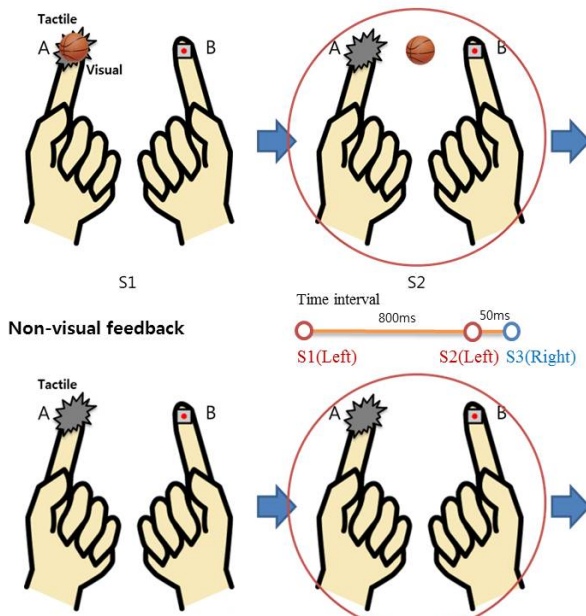

S1
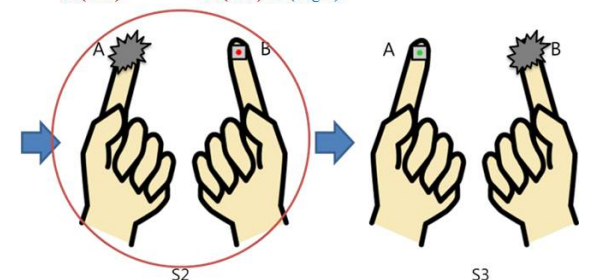

S3

Fig. 10. Administering for the saltation effect with three timed vibro-tactile stimulations with visual effects (above) and without (below)

sensation and 6 repetitions) +12 times without visual feedback ( 2 directions and 6 repetitions). Each stimulation was followed by a 10 second inter-stimulus rest interval. As it was so in the first experiment, for each treatment condition, two exact same stimulation patterns were given, then subjects were asked to indicate the place of phantom sensations in terms of the five prescribed positions (L1 L5). The subjects were explicitly asked to report the place of tactile sensation (e.g. rather than visual) right after experiencing the stimulation. In addition, after all trials, they were asked to answer a short survey about their subject feelings (questions shown in Table 4). The fifth survey question asked of the perception regarding the directionality.

Table 4. The five survey questions for Experiment II regarding the subjective feel for the phantom sensation answered in 7 Likert scale

\begin{tabular}{|l|l|}
\hline Q1 & $\begin{array}{l}\text { Were you able to perceive phantom sensation? } \\
(1: \text { Not at all } \sim 7: \text { Very well })\end{array}$ \\
\hline Q2 & $\begin{array}{l}\text { When you perceive phantom sensation, did visual feedback affect you? } \\
(1: \text { Not at all } \sim 7: \text { Very much })\end{array}$ \\
\hline Q3 & $\begin{array}{l}\text { How confidence are you about your answer to Q1? } \\
(1: \text { Not confident at all 7: Very confident })\end{array}$ \\
\hline Q4 & $\begin{array}{l}\text { How long did it take you to perceive the phantom sensation if any? } \\
(1: \text { Instantly } ~ 7: \text { Few seconds })\end{array}$ \\
\hline Q5 & $\begin{array}{l}\text { Were you able to recognize a particular direction? } \\
(1: \text { Not at all } ~ 7: \text { Very well })\end{array}$ \\
\hline
\end{tabular}




\subsection{Results}

Table 5 shows that the sensed tactile locations after saltation were statistically different from the actual location of stimulation for both when visual effect was given and, surprisingly again, when not given at all. Note that, according to Miyazaki, phantom sensation from saltation was not observed without visual feedback nor mediating object [27]. While our result is somewhat contrary, its extent was very small (see Figure 11(b)).

Table 5. Effects of saltation. Perceived location for S2 were in all cases different from S1 (both $\mathrm{S} 1$ and S2 stimluations were given at the same physical location) with statistical significance ( $p$-values) indicating the existence of the saltation effect.

\begin{tabular}{|l|l|l|}
\hline $\begin{array}{l}\text { Intended position } \\
\text { (with visual) }\end{array}$ & $\begin{array}{l}\text { p-value for difference } \\
\text { between S1 and S2 } \\
\text { (Stim. Dir. L } \rightarrow \text { R) }\end{array}$ & $\begin{array}{l}\text { p-value for difference } \\
\text { between S1 and S2 } \\
\text { (Stim. Dir. } \mathrm{R} \rightarrow \mathrm{L} \text { ) }\end{array}$ \\
\hline P1 & $<\mathbf{0 . 0 0 1}$ & $<\mathbf{0 . 0 0 1}$ \\
P2 & $<\mathbf{0 . 0 0 1}$ & $<\mathbf{0 . 0 0 1}$ \\
P3 & $<\mathbf{0 . 0 0 1}$ & $<\mathbf{0 . 0 0 1}$ \\
P4 & $<\mathbf{0 . 0 0 1}$ & $<\mathbf{0 . 0 0 1}$ \\
P5 & $<\mathbf{0 . 0 0 1}$ & $<\mathbf{0 . 0 0 1}$ \\
\hline
\end{tabular}

Figure 11(a) and (b) each shows the locations of the tactile sensation (vertical axis), elicited by saltation, as reported by the users vs. the intended locations (horizontal axis) of sensation with visual effects and without. When visual feedback was given, there were as usual five intended location of sensation according to the five respective locations of the visual feedback. However, only four statistically different sensed locations for S2 were found (i.e. L1, L2, L3, L4=L5; p-values L1-L2: <0.001; L2-L3: <0.001; L3-L4: <0.001; L4-L5: 0.088). There were significant differences in the accuracy (or variance) for the intermediate locations, between L3 L5 and P3 P5. Higher variance and lower accuracy/match (even at L1/P1) was observed as compared to the case of funneling, getting worse at the place of the third stimulation. When there was no visual feedback, we were only looking for (if it existed) one location of phantom sensation somewhere between the fingers. As mentioned above, this nonvisual case did exhibit a phantom sensation at one location significantly different, though very small, from the place of actual stimulation, around $0.83 \mathrm{~cm}$ away. Postbriefings with the subjects also reflected the observation this sensation was barely perceivable. 


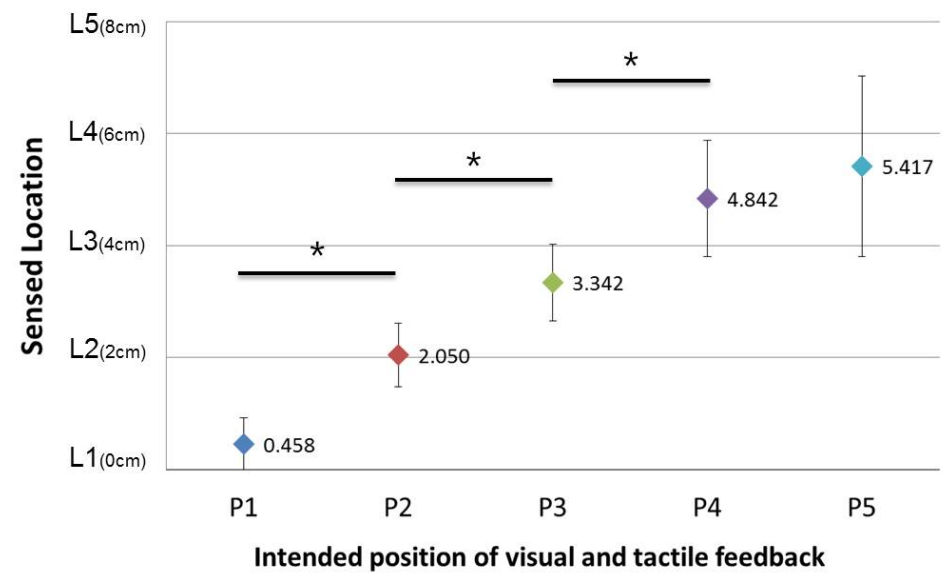

(a) With the associated visual effects

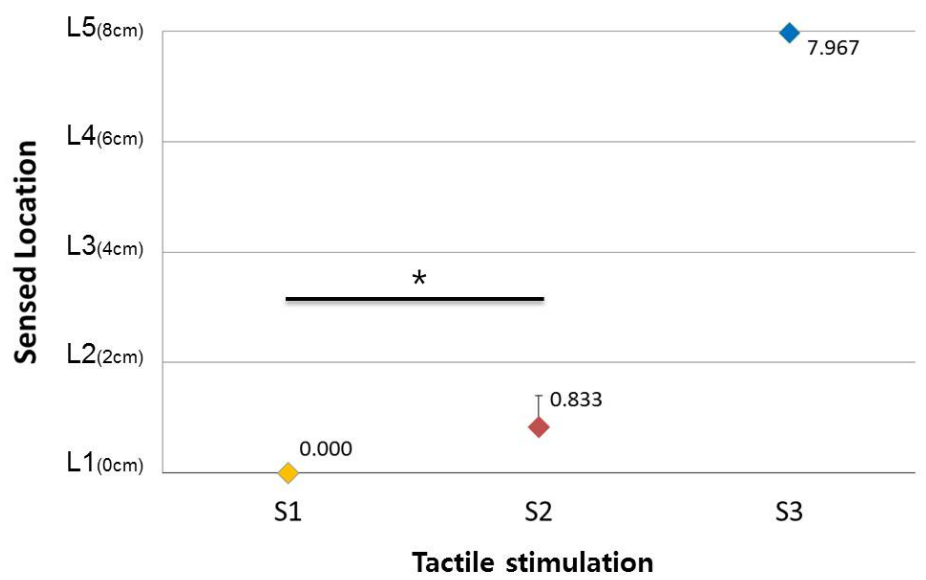

(b) Without visual effects

Fig. 11. Accuracy of reported locations of the phantom sensation with respect to the intended for saltation: (a) with associated visual effect and (b) without. Star marks indicate those with statistically significant differences.

Finally Figure 12 shows the responses to the five survey questions, which are consistent with the quantitative analysis. Subjects were conscious of the helpful effects of the visual feedback and confident of their phantom sensations. Subjects were also able to recognize the direction of the stimulation and the described the sensation to as soft bounce (15/20), hard contact (3/20) and moving vibration $(2 / 20)$ in the post-briefing. 


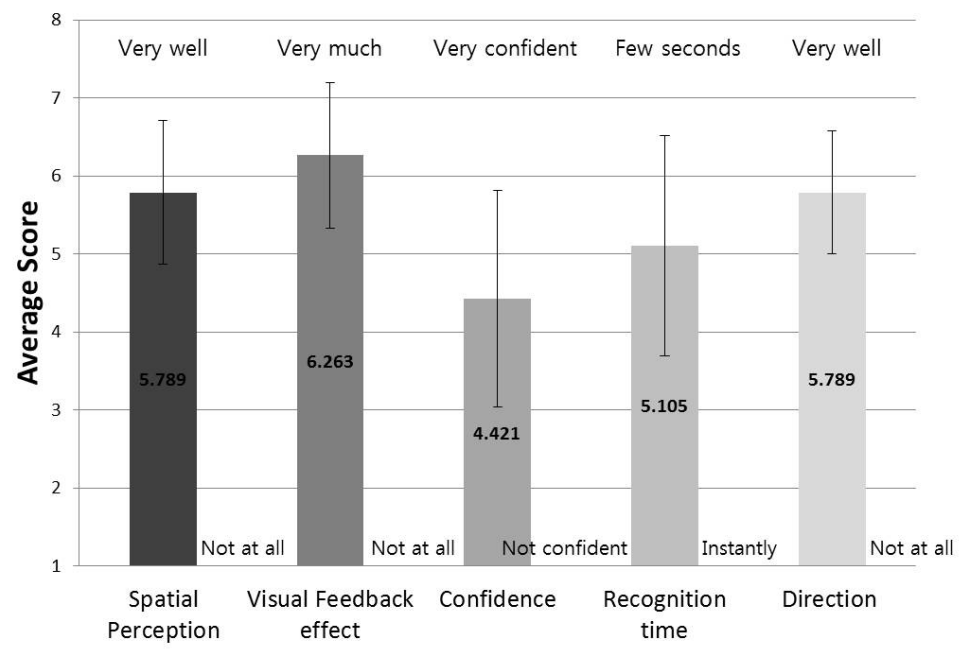

Fig. 12. The collective responses to the general usability/experience survey

\section{Discussion and Conclusion}

Previous research has found the phantom tactile sensations for virtual objects external to one's body. However, it required a virtual imagery to be attached to the user for directly extending one's body. This paper has investigated in whether similar phantom tactile sensations exist when the virtual object is visually detached from the user's body. Our results have shown that in addition to the perception of the phantom sensations with the "detached" visual feedback, the interaction experience was significantly enriched (vs. when without explicit visual feedback). We also discovered for the first time that for funneling, phantom sensations can be elicited without any visual feedback at all. We can further conjecture with both mediating visual feedback and the actual dynamic visual content, the tactile experience has to be improved even more with even higher localization controllability.

While the two tested phantom sensation techniques generated similar qualitatively enhanced tactile experience (both quantitatively and qualitatively), the funneling technique produced higher overall accuracy than saltation. On the other hand, saltation due to its nature seems fitting as a mean to provide directional tactile experience. The post-briefing also revealed the same. While both subjects answered both techniques did produce phantom sensations for certain, they also felt the funneling to have produced more efficient and stronger sensation.

The findings can be applied to the tactile interaction design using minimal number of actuators on a variety of media platforms including the mobile, holography and augmented reality. From a more practical perspective, we would be much interested in comparing the relative effect between (1) the usual single vibrator scheme and (2) using perhaps 2 or 4 vibrators for 1D or 2D [22] localized phantom tactile effect. The single vibrator scheme simply cannot be used for the case of "Out of the Body" 
virtual objects with virtual medium (e.g. two handed interaction with a dynamic holographic object). However, for the case of "Out of the Body" virtual objects with physical medium (e.g. two handed interaction with moving virtual objects on a mobile device), it is unclear whether there is sufficient benefit-to-cost in the additional effort to employ funneling or saltation. Single vibrator scheme has been quite successful in eliciting pseudo-haptic effects in smart phones and game controllers [8].

However the distinction must be made clear in terms of the role of the visual feedback. The single vibrator scheme can be explained to be a phantom or pseudo sensation directly caused by the visual feedback, while our paper has addressed the phantom sensation being strengthened reversely by the visual effect. Note that our performance measures were tactile experience, and not visual. This suggests, that although our experiment has only tested the case with virtual medium, it is plausible to expect that the tactile experience to be significantly richer than the case of single vibrator scheme, e.g. if applied to mobile devices. The plausibility is high also from the previous research indicating the weakened sensation when the "connecting" medium was virtual [24]. Either way, the combined effect can be explained by the Modality Competition Theory that the modal fusion will depend on the disparity among the stimulations in term of their consistency [33, 41]. For instance, the disparity between the location of the phantom sensation and the visual feedback seems less than that that in a single vibrator scheme.

In fact, our next focus will be to verify such a proposition. In addition, we are interested in the neurological or cognitive explanation to this phenomenon. The typical explanation for funneling and saltation has been based on the continuity of the body map in the somato-sensory area in the brain [9]. However, with the discovery of the "Out of the Body" phenomenon, it seems there may be a cognitive element to it too (reconfiguring of the body map is also possible but it is usually regarded a very slow process as demonstrated in the phantom limbs [33]).

Acknowledgement. This work was supported by the National Research Foundation of Korea (NRF) grant funded by the Korea government (MEST) (No 2012-0009232) and this research was funded by the Forensic Research Program of the National Forensic Service (NFS), Ministry of Security and Public Administration, Korea.

\section{References}

1. Alles, D.S.: Information transmission by phantom sensations. IEEE Transactions on ManMachine Systems 11(1), 85-91 (1970)

2. Arduino, http: //www . arduino.cc/

3. Barghout, A., Kammerl, J., Jongeun, C., Steinbach, E., Saddik, A.E.: Spatial resolution of vibrotactile perception on the human forearm when exploiting funneling illusion. In: IEEE International Workshop on HAVE (2009)

4. Bekesy, G.V.: Funneling in the nervous system and its role in loudness and sensation intensity on the skin. Journal of the Acoustical Society of America, 399-412 (1958)

5. Bolt, R.A.: Put-That-There, Voice and Gesture at the Graphics Interface. Computer Graphics, 262-270 (1980) 
6. Borst, C.W., Baiyya, V.B.: Enhancing VR-based Visualization with a 2D Vibrotactile Array. In: Virtual Reality Software and Technology. ACM (2007)

7. Borst, C.W., Cavanaugh, C.D.: Haptic Controller Design and Palm-sized Vibrotactile Array. Technical Report, Center for Advanced Computer Studies, University of Louisiana, Lafayette (2004)

8. Bouncing Ball on Nokia N900, http: / / bit.1y/x2-bouncingball/

9. Chen, L.M., Friedman, R.M., Roe, A.W.: Optical imaging of a tactile illusion in area $3 \mathrm{~b}$ of the primary somatosensory cortex. Science 302(5646), 881-885 (2003)

10. Cholewiak, R.W., Collins, A.A.: The generation of vibro-tactile patterns on a linear array: Influences of body site, space, and time. Perception \& Psychophysics, 1220-1235 (2000)

11. Corradini, A., Wesson, R.M., Cohen, P.R.: A map-based system using speech and 3D gestures for pervasive computing. In: 4th IEEE International Conference on Multimodal Interfaces, pp. 191-196 (2002)

12. Eimer, M., Forster, B., Vibell, J.: Cutaneous saltation within and across arms: A new measure of the saltation illusion in somatosensation. Percept. Psychophys. 67(3), 458-468 (2005)

13. Flach, R., Haggard, P.: The cutaneous rabbit revisited. Journal of Experimental Psychology: Human Perception and Performance, 717-732 (2006)

14. Geldard, F.A., Sherrick, C.E.: The Cutaneous Rabbit: A perceptual Illusion. Science, 178-179 (1972)

15. Geldard, F.A.: Saltation in Somesthesis. Psychological Bulletin, 136-175 (1982)

16. Geldard, F.A.: Sensory Saltation: Metastability in the Perceptual World. Lawrence Erlbaum Associates, Hillsdale (1975)

17. Grasso, M.A., Ebert, D.S., Finin, T.W.: The integrality of speech in multimodal interfaces. ACM Transactions on Computer-Human Interaction (1998)

18. Hoggan, E., Anwar, S., Brewster, S.: Mobile multi-actuator tactile displays. In: Oakley, I., Brewster, S. (eds.) HAID 2007. LNCS, vol. 4813, pp. 22-33. Springer, Heidelberg (2007)

19. Israr, A., Poupyrev, I.: Tactile Brush: Drawing on Skin with a Tactile Grid Display. In: ACM CHI, pp. 2019-2028 (2011)

20. Jung, J., Choi, S.: Perceived magnitude and power consumption of vibration feedback in mobile devices. In: Jacko, J.A. (ed.) HCI 2007. LNCS, vol. 4551, pp. 354-363. Springer, Heidelberg (2007)

21. Kilgard, M.P., Merzenich, M.: Anticipated stimuli across skin. Nature, 663 (1995)

22. Kim, Y., Lee, J., Kim, G.: Extending out of the body saltation to 2D mobile tactile interaction. In: 10th Asia Pacific Conference on Computer Human Interaction, pp. 67-74 (2012)

23. Lee, J., Spence, C.: Assessing the benefits of multimodal feedback on dual-task performance under demanding conditions. In: 22nd British HCI Group Annual Conference on HCI, pp. 185-192 (2008)

24. Lee, J., Kim, Y., Kim, G.J.: Funneling and Saltation Effects for Tactile Interaction with Virtual Objects. In: ACM CHI, pp. 3141-3148 (2012)

25. McGurk, H., MacDonald, J.W.: Hearing Lips and Seeing Voices. Nature (1976)

26. Mine, M.R., Brooks Jr., F.P., Sequin, C.H.: Moving Objects in Space: Exploiting Proprioception in Virtual-Environment Interaction. In: ACM SIGGRAPH, pp. 19-26 (1997)

27. Miyazaki, M., Hirashima, M., Nozaki, D.: The "Cutaneous Rabbit" hopping out of the body. The Journal of Neuroscience 30(5), 1856-1860 (2010)

28. Mizukami, Y., Sawada, H.: Tactile information transmission by apparent movement phenomenon using shape-memory alloy device. International Journal on Disability and $\mathrm{Hu}-$ man Development, 277-284 (2006) 
29. ARToolKit For OpenSceneGraph(OSGART), http: / / www . osgart . org/

30. Oviatt, S.L.: Multimodal interfaces. In: The Human-Computer Interaction Handbook: Fundamentals, Evolving Technologies and Emerging Applications, pp. 286-304 (2003)

31. Rahal, L., Cha, J., Kammerl, J., El Saddik, A., Steinbach, E.: Investigating the influence of temporal intensity changes on the apparent movement phenomenon. In: Virtual Environments, Human-Computer Interfaces and Measurement Systems, pp. 310-313 (2009)

32. Raisamo, J., Raisamo, R., Surakka, V.: Evaluating the effect of temporal parameters for vibrotactile saltatory patterns. In: International Conference on Multimodal interfaces, pp. 317-326 (2009)

33. Ramachandran, V.S., Blakeslee, S.: Phantoms in the Brain. Harper Collins Publishers (1998)

34. Richard, P., Burdea, G., Gomez, D., Coiffet, P.: A comparison of Haptic, Visual and Auditive Force Feedback for Deformable Virtual Objects. In: ICAT Conference (1994)

35. Richter, H., Hang, A., Blaha, B.: The PhantomStation: Towards funneling remote tactile feedback on interactive surfaces. In: Augmented Human International Conference (2011)

36. Sherrick, C.E.: A scale for rate of tactual vibration. Journal of the Acoustical Society of America (1985)

37. Sherrick, C.E., Cholewiak, R.W., Collins, A.A.: The localization of low-and high frequency vibrotactile stimuli. Journal of the Acoustical Society of America 169 (1990)

38. Tan, H.Z., Pentland, A.: Tactual displays for wearable computing. IEEE Personal and Ubiquitous Computing 1(4), 84-89 (1997)

39. Waller, D.: Factors Affecting the Perception of Inter Object Distances in Virtual Environments. Presence: Teleoperators and Virtual Environments, 657-670 (1999)

40. Warren, J.P., Santello, M., Tillery, S.I.H.: Electrotactile stimuli delivered across fingertips inducing the Cutaneous Rabbit Effect. Exp. Brain Res., 419-426 (2010)

41. Wickens, C.D., Sandry, D.L., Vidulich, M.: Compatibility and resource competition between modalities of input, central processing, and output. Human Factors 25(2), 227-248 (1983) 